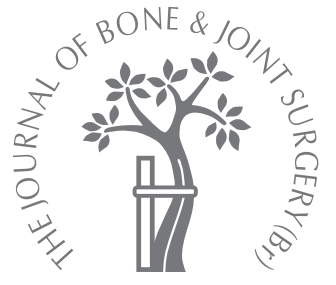

J. Wilson,

T. J. Bonner,

M. Head,

J. Fordham,

S. Brealey,

A. Rangan

From The James

Cook University

Hospital,

Middlesbrough,

England

J. Wilson, MRCS, MRCS(Ed) Registrar

T. J. Bonner, MRCS,

Specialist Registrar

M. Head, MRCS, Specialist

Registrar

I. J. Fordham, FRCP, Consultant

Rheumatologist

A. Rangan, FRCS(Ed)

Consultant

Department of Trauma and

Orthopaedics

The James Cook University

Hospital, Marton Road,

Middlesbrough, Teesside TS3

3BW, UK.

S. Brealey, BSc, $\mathrm{PhD}$

Research Fellow

Department of Health Sciences

Second Floor (Area 4),

Seebohm Rowntree Building,

University of York, Heslington,

York YO10 5DD, UK.

Correspondence should be sent to $\mathrm{Mr} \mathrm{T}$. J. Bonner; e-mail:

timbonner@doctors.org.uk

(C)2009 British Editorial Society of Bone and Joint Surgery doi:10.1302/0301-620X.91B6. $22346 \$ 2.00$

$J$ Bone Joint Surg [Br]

2009;91-B:772-5.

Received 26 January 2009.

Accepted 12 March 2009

\title{
Variation in bone mineral density by anatomical site in patients with proximal humeral fractures
}

Low-energy fractures of the proximal humerus indicate osteoporosis and it is important to direct treatment to this group of patients who are at high risk of further fracture. Data were prospectively collected from 79 patients ( 11 men, 68 women) with a mean age of 69 years (55 to 86) with fractures of the proximal humerus in order to determine if current guidelines on the measurement of the bone mineral density at the hip and lumbar spine were adequate to stratify the risk and to guide the treatment of osteoporosis. Bone mineral density measurements were made by dual-energy $x$-ray absorptiometry at the proximal femur, lumbar spine (L2-4) and contralateral distal radius, and the T-scores were generated for comparison. Data were also collected on the use of steroids, smoking, the use of alcohol, hand dominance and comorbidity.

The mean T-score for the distal radius was -2.97 (SD 1.56) compared with -1.61 (SD 1.62) for the lumbar spine and $\mathbf{- 1 . 7 8}$ (SD 1.33) for the femur. There was a significant difference between the mean lumbar and radial $T$ scores (1.36 (1.03 to 1.68$) ; p<0.001$ ) and between the mean femoral and radial T-scores (1.18 (0.92 to 1.44); $p<0.001)$. The inclusion of all three sites in the determination of the T-score increased the sensitivity to $66 \%$ compared with that of $46 \%$ when only the proximal femur and lumbar spine were used. This difference between measurements in the upper limb compared with the axial skeleton and lower limb suggests that basing risk assessment and treatment on only the bone mineral density taken at the hip or lumbar spine may misrepresent the extent of osteoporosis in the upper limb and the subsequent risk of fracture at this site.

The assessment of osteoporosis must include measurement of the bone mineral density at the distal radius to avoid underestimation of osteoporosis in the upper limb.

Osteoporosis is the most common metabolic bone disease in the western world and is recognised as a major cause of disability and morbidity in older men and women. ${ }^{1,2}$ It has been described as the silent epidemic and manifests clinically as fragility fractures predominantly of the distal radius, proximal femur and spine. ${ }^{3}$ Research and treatment of fragility fractures have commonly focused on proximal femoral fractures because they represent the most dramatic consequence of the disease, and by their very nature demand urgent treatment. ${ }^{1}$ However, fragility fractures of the humerus, forearm and wrist account for one-third of the total incidence of fractures in the elderly. ${ }^{4-6}$ It may be argued that a fracture of the upper limb is less limiting because it does not prevent walking, but it can play a considerable role in depriving patients of their independence. ${ }^{1}$ This is usually temporary but in $6 \%$ of cases may lead to a permanent move to a nursing home. ${ }^{1}$ Fractures of the upper limb also demand considerable allocation of resources. ${ }^{1}$ Onethird of such fractures require operative intervention and $37 \%$ in-patient rehabilitation. ${ }^{1}$ The median length of hospital stay for these injuries is 46 days. ${ }^{1}$ Furthermore, fractures of the proximal humerus are associated with more prolonged in-patient rehabilitation than any other fracture of the upper limb.

The World Health Organisation (WHO $)^{8}$ defines osteoporosis by comparing the bone mineral density (BMD) with that of a gendermatched, healthy young adult reference population. The T-score indicates the difference between the BMD of an individual and the mean score of the reference population. It is measured in SD units above or below the mean. The WHO 'gold standard' for diagnosing osteoporosis in women is a T-score of less than -2.5 at any one of three skeletal sites, the femur, lumbar spine or distal radius. ${ }^{8}$ The most common clinical method of assessing the BMD is dual-energy $\mathrm{x}$-ray absorptiometry (DXA) of 
Table I. The incidence of risk factors for osteoporosis in the sample group

\begin{tabular}{lc}
\hline & Frequency (\%) \\
\hline Current smoker & $14(17.7)$ \\
Alcohol use & $19(24.1)$ \\
Excess alcohol (>21 units per week) & $1(1.3)$ \\
Oral steroid use & $7(8.9)$ \\
Diabetes & $15(19.0)$ \\
Dominant side fractured & $39(49.4)$ \\
\hline
\end{tabular}

the central skeleton, with measurements taken at the hip and lumbar spine. ${ }^{9}$

We measured the BMD at the hip, lumbar spine and distal radius, of men and women with fractures of the humerus in order to assess the sensitivity of these sites in diagnosing osteoporosis in accordance with WHO criteria.

\section{Patients and Methods}

The series included 79 consecutive patients (11 men, 68 women) with a mean age of 69 years (55 to 86) who presented to a fracture clinic or were admitted to hospital after sustaining a fracture of the proximal humerus and who were referred for assessment of their BMD between October 2000 and March 2002. Our hospital is the only one managing trauma for a population of approximately 340 000. Patients in whom treatment for osteoporosis had already been initiated were not referred for BMD assessment and therefore excluded from the study. All low-energy fractures were included in the study. These were defined as a fall from a standing height or lower.

Information on hand dominance, smoking status, the use of steroids, medical history and age was collected. The DXA scans were performed with BMD measurements taken at the standard positions at the femoral neck, lumbar spine (L2-4) and at the contralateral distal radius, using the Lunar DPXL bone densitometer (Lunar Corporation, Madison, Wisconsin). This equipment was calibrated by daily quality assurance checks and weekly control checks using a phantom spine. These values were then converted to T-scores using the United Kingdom reference data provided by the manufacturer.

The information was anonymised and recorded on an Excel database (Microsoft, Redmond, Washington). If osteoporosis was diagnosed according to the $\mathrm{WHO}$ criteria, the patient was offered treatment and follow-up according to local policy.

Statistical analysis. This was performed using SPSS version 14 (SPSS Inc, Chicago, Illinois). The mean T-scores were compared using two-tailed, paired-sample $t$-tests and a p-value $\leq 0.01$ was deemed to be significant.

\section{Results}

Other baseline characteristics and risk factors for osteoporosis in the study group are shown in Table I which illustrates that the patients had many of the expected risk factors for
Table II. Mean (SD) T-scores for the bone mineral density at different skeletal sites for men and women

\begin{tabular}{llll}
\hline & Male & Female & Total \\
\hline Age in yrs (range) & $68(55$ to 86$)$ & $69(55$ to 85$)$ & 69 (55 to 86) \\
& & & \\
T-score & & & \\
$\quad$ Distal radius & $-2.56(1.52)$ & $-3.03(1.56)$ & $-2.97(1.56)$ \\
$\quad$ Lumbar spine & $-1.41(1.20)$ & $-1.64(1.69)$ & $-1.61(1.62)$ \\
Femur & $-2.00(0.93)$ & $-1.74(1.39)$ & $-1.78(1.33)$ \\
\hline
\end{tabular}

osteoporosis. The key results for the BMD in men and women within this sample are shown in Table II. They show that there was a significant difference between the T-scores at the separate body locations at which the BMD was measured. The mean T-score for the distal radius was -2.97 (SD 1.56), for the lumbar spine -1.61 (SD 1.62) and the femur 1.78 (SD 1.33). There was a significant difference between the mean lumbar and radial T-scores (1.36 (1.03 to 1.68), $t$ test, $\mathrm{p}<0.001)$ and between the mean femoral and radial Tscores $(1.18$ (0.92 to 1.44$) t$-test, $\mathrm{p}<0.001)$.

There were clear differences in the identification of osteoporosis at the different anatomical sites. Only $36(45.6 \%)$ of the patients would have been diagnosed with osteoporosis if the BMD had been measured at the lumbar spine and proximal femur. When the BMD of the distal radius was measured alone, 46 patients $(58.2 \%)$ fulfilled the WHO diagnostic criteria. Furthermore, when the measurements at all three sites were considered together, $52(65.8 \%)$ patients fulfilled the criteria for osteoporosis.

The confidence intervals (CI) for three estimates of sensitivity were calculated. ${ }^{10}$ They showed that for the combination of the lumbar spine and femur the sensitivity was $46 \%$ (95\% CI 35 to 57), for the radius alone $58 \%$ (47 to 68 ) and for all three sites $66 \%$ (55 to 75 ). Therefore the difference between the sensitivity of using measurements based on the femur and lumbar spine compared with that using all three sites was significant at the $5 \%$ level because the upper CI for the lumbar spine and femur did not cross the estimate of the sensitivity for all three sites.

\section{Discussion}

The incidence of osteoporosis and fragility fractures is increasing. The risk of osteoporosis increases with age and particularly affects post-menopausal women. The Scottish Needs Assessment Programme ${ }^{11}$ estimated that one in three women will suffer a fragility fracture after the age of 50 years. Dennison, Cole and Cooper ${ }^{12}$ projected that the current world prevalence of 323 million cases will rise to 1.55 billion by the year 2050. Based on current trends, by 2016 the annual incidence of hip fractures in the United Kingdom will have risen to 117000 compared with 46000 in 1985. The annual cost of treating osteoporotic fractures in the United Kingdom is presently $£ 1.7$ billion and is expected to rise further. ${ }^{13}$ 
Dual-energy x-ray absorptiometry is currently the most accurate technique for measuring BMD. ${ }^{14,15}$ Our study has shown that there is wide variation in the BMD when measured at different skeletal sites. When considering injuries to the upper limb, the BMD measured at the distal radius may represent the risk of osteoporotic humeral fractures better than that measured at the axial skeleton or lower limbs.

Arlot et $\mathrm{al}^{16}$ in their study of 725 post-menopausal women without a history of fracture also found substantial variation in measurements of the BMD at different sites. They showed that T-scores at the distal radius were lower than those at the hip and lumbar spine and that measurements at the distal radius yielded the highest proportion of women reaching the WHO criteria for osteoporosis.

In current United Kingdom medical practice the central skeleton is preferred for measuring the BMD. ${ }^{9}$ The main reasons for this are that BMD at the hip is the most reliable predictor of the risk of fracture of the hip and that at the lumbar spine is the optimal site for monitoring the response to treatment. ${ }^{17-21}$ However, the lumbar spine is commonly affected by degenerative changes which can increase the apparent BMD. ${ }^{9}$ The distal radius has a large proportion of cancellous bone which, when compared with cortical bone, is affected to a greater extent by age-related changes in the BMD. Therefore it may offer an easily accessible site for assessing the response to treatment. ${ }^{15}$

Ito et $\mathrm{a}^{22}$ studied different sites and measurement techniques to determine the best investigation to quantify changes in the BMD in response to treatment. The distal radius was useful as a site for monitoring changes in the BMD and had long-term precision comparable with that of the spine. However, this was only when the BMD at the distal radius was assessed by quantitative CT. In an epidemiological study of 651 healthy men and women, Jørgensen et $\mathrm{al}^{2}$ measured the BMD at different skeletal sites using DXA and ultrasonography. The BMD at the femoral neck was used as the indicator of osteoporosis. Further analysis showed that the BMD at the distal forearm had a sensitivity of $63 \%$ and a specificity of $96 \%$ for predicting osteoporosis at the hip. However, these results may be interpreted with some caution because the WHO definition of osteoporosis included the presence of a T-score of -2.5 or less at any of the sites i.e., distal radius, hip or lumbar spine.

Our study was not longitudinal and therefore we cannot comment on the risk of subsequent fractures generally or specifically in the upper limb. Also, all fracture configurations of the proximal humerus were included. It may be argued that not all types of fracture are associated with osteoporosis, but we took the pragmatic view that all lowenergy injuries should be included.

Our study showed that low-energy fractures of the proximal humerus are more closely associated with a low BMD at the distal radius than with a low BMD at the hip, lumbar spine or a combination of the hip and lumbar spine. This has not been demonstrated previously. It is well known that local measurements at the site of a possible fracture are much more predictive of the fracture than are measurements elsewhere in the skeleton. This has been shown for fractures of the hip, lumbar spine and forearm. ${ }^{23}$ The reason for treating osteoporosis is to prevent fractures. Therefore identification of patients at greatest risk allows treatment to be directed to those who will benefit the most. Clowes et $\mathrm{a}^{23}$ applied multivariate analysis to their data and found that if a peripheral measurement was combined with a total hip DXA measurement, the odds ratio for predicting a subsequent fracture was increased. Therefore in order to improve the cost-effectiveness of treatment and to target those most likely to benefit, central and peripheral measurements should be used at least for diagnostic purposes if not to monitor the response to treatment.

For patients who have sustained a fragility fracture the results re-emphasise that the risk of fracture is only partially explained by a low BMD and that other factors are important. Even when all three BMD measurements were included, only $66 \%$ reached the WHO criteria. Further research to improve diagnostic tools is necessary. For patients who have not yet sustained a fragility fracture it shows that changes in the BMD are not uniform and that assessment of the BMD at the distal radius should not be neglected.

In conclusion, our study has highlighted the regional variation in BMD and that measurements of the BMD in the upper limb are significantly lower than those in the lumbar spine and femur in patients with fractures of the proximal humerus. Measurement of the BMD at the hip and lumbar spine, if used alone may underestimate the risk of osteoporosis in the upper limb. Measurement of the BMD at the radius should not be omitted when osteoporosis is being investigated.

\section{Supplementary material}

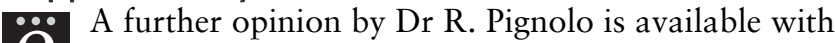
the electronic version of this article on our website at www.jbjs.org.uk

No benefits in any form have been received or will be received from a commercial party related directly or indirectly to the subject of this article.

\section{References}

1. Lübbeke A, Stern R, Grab B, et al. Upper extremity fractures in the elderly: consequences on utilization of rehabilitation care. Aging Clin Exp Res 2005;17:276-80.

2. Jørgensen HL, Warming L, Bjarnson NH, Andersen PB, Hassager C. How does quantitative ultrasound compare to dual $x$-ray absorptiometry at various skeletal sites in relation to the WHO diagnosis categories? Clin Physio/ 2001;21:51-9.

3. Cummings DT, Kelsey JL, Nevitt MC, O'Dowd KJ. Epidemiology of osteoporosis and osteoporotic fractures. Epidemiol Rev 1985;7:178-208.

4. Lee SH, Dargent-Molina P, Breart G. Risk factors for fractures of the proximal humerus: results from the EPIDOS prospective study. J Bone Miner Res 2002;17:81725

5. Nguyen TV, Center JR, Sambrook PN, Eisman JA. Risk factors for proximal humerus, forearm and wrist fractures in elderly men and women: the dubbo osteoporosis epidemiology study. Am J Epidemio/ 2001;153:587-95.

6. Seeley D, Browner W, Nevitt M, et al. Which fractures are associated with low appendicular bone mass in elderly women? Ann Intern Med 1991;115:837-42.

7. Lippuneer K, von Overbeck J, Perrelet R, Bosshard H, Jaeger P. Incidence and direct medical costs of hospitalisations due to osteoporotic fractures in Switzerland. Osteoporos Int 1997;7:414-25. 
8. WHO Study Group. Assessment of fracture risk and its application to screening for postmenopausal osteoporosis. Geneva: WHO, 1994: Technical report series 843.

9. Blake GM, Fogelman I. The role of DXA bone density scans on the diagnosis and treatment of osteoporosis. Postgrad Med J 2007;83:509-17.

10. Wilson EB. Probable inference, the law of succession, and statistical inference. $J$ Am Stat Assoc 1927;22:209-12.

11. The Scottish Needs Assessment Programme. Osteoporosis. Glasgow: Scottish Forum for Public Health Medicine. 1997:1-47.

12. Dennison E, Cole Z, Cooper C. Diagnosis and epidemiology of osteoporosis. Curr Opin Rheumatol 2005;17:456-61.

13. Torgerson D, Bell-Syer S. Hormone replacement therapy and prevention of nonvertebral fractures: a meta-analysis of randomised trials. JAMA 2001;285:2891-7.

14. Ravn P, Hetland ML, Overgaard K, Christiansen C. Pre-menopausal and post menopausal changes in bone mineral density of the proximal femur measured by dual-energy x-ray absorptiometry. J Bone Miner Res 1994;9:1975-80.

15. Genant HK, Steiger P, Faulkner KG, et al. Non-invasive bone mineral analysis: recent advances and future directions. In: Christiansen C, Overgaard K, eds. Osteoporosis, Third international symposium on Osteoporosis. Vol. 2. Copenhagen: Osteopress, 1990:435-41.
16. Arlot ME, Sornay-Rendu E, Garnero P, Vey-Marty B, Delmas PD. Apparent preand postmenopausal bone loss evaluated by DXA at different skeletal sites in women: the OFLEY cohort. J Bone Miner Res 1997;12:683-90.

17. Marshall D, Johnell $\mathbf{0}$, Wedel H. Meta-analysis of how well measures of bone mineral density predict occurrences of osteoporotic fractures. BMJ 1996;312:1254-9.

18. Stone KL, Seeley DG, Lui L-Y, et al. BMD at multiple sites and risk of fracture of multiple types: long-term results from the study of osteoporotic fractures. $J$ Bone Miner Res 2003;18:1947-54

19. Johnell O, Kanis JA, Oden A, et al. Predictive value of BMD for the hip and other fractures. J Bone Miner Res 2005;20:1185-94.

20. Eastell R. Treatment of postmenopausal osteoporosis. N Engl J Med 1998;338:736-46.

21. Gluer CC. Monitoring skeletal change by radiological techniques. J Bone Miner Res 1999;14:1952-62.

22. Ito M, Nishida A, Kono J, et al. Which bone densitometry and which skeletal site are clinically useful for monitoring bone mass? Osteoporos Int 2003;14:959-64.

23. Clowes JA, Eastell R, Peel NF. The discriminative ability of peripheral and axial bone measurements to identify proximal femoral, vertebral, distal forearm and proximal humeral fractures: a case control study. Osteoporos Int 2005;16:1794-802. 